\title{
SIMULATION SOFTWARE TOOL TO EVALUATE INTERFERENCES BETWEEN CELLULAR PUBLIC NETWORKS AND GSM-R SYSTEM
}

\author{
M. GONZÁLEZ-GONZALO, J. CALLE-SÁNCHEZ, P.C. GUTIÉRREZ \& J.I. ALONSO \\ Dpto. Señales, Sistemas y Radiocomunicaciones, E.T.S.I. Telecomunicación. \\ Universidad Politécnica de Madrid, Avenida Complutense, 30, 28040, Madrid.
}

\begin{abstract}
Railway scenarios present several specific features that difficult the deployment of a radio communication network, especially for high-speed environments. Railway passenger's demand for high capacity communication systems for personal purposes is continuously growing. This demand drives the installation of public commercial communication systems: 3G, LTE, which not only coexist next to GSM-R system infrastructure, but may be also deployed in frequency bands which lie next to GSM-R ones. This fact may lead to interference issues between both systems, as reported by several railway operators.

Regarding the critical nature of railway communication systems, it is advocated the avoidance of interference issues in advance. However, this process is a complex task that must be addressed with specific software tools that takes into account the specificities of high-speed railway communication systems. In this paper, the authors present a novel software tool designed for the evaluation of coexistence between high-speed railway communication systems and public commercial ones.

Keywords: GSM-R, high-speed railway, interferences, LTE, railway environments, software tool.
\end{abstract}

\section{INTRODUCTION}

Nowadays the number of high-speed on-board train users demanding broadband data services, is increasing due to the high penetration rate of smartphones, tablets, and laptops. Railway operators and telecommunication companies have proven to be interested in providing broadband access to train passengers. However, railway environments are hard environments for communications, due to factors such as propagation, fast changes in the channel due to high speeds, etc.

In order to overcome these constraints and to reduce deployment infrastructure costs, telecommunications companies usually co-locate commercial systems in the same places where GSM-R ones are deployed. The co-location of cellular commercial systems in the same sites as GSM-R system, may lead to interference issues if both kinds of systems are deployed in adjacent frequency bands [1-3].

Besides, the potential interference issues gain in importance regarding the spectrum digital dividend process, where new broadband systems like LTE can be deployed in the $800 \mathrm{MHz}$ band. When assessing coexistences between LTE and GSM-R, it is important to remark that railway industry is considering the migration towards new broadband and reliable communication system, like LTE [4]. It is expected that for a midterm scenario, both systems will coexist in railway environments, leading to harmful interference situations.

In this line, several researches have suggested the possibility of interferences into GSM-R from LTE networks like in $[5,6]$.

Under this scope, it is important to perform an assessment process on coexistence between commercial communication systems and GSM-R. The outcome of this assessment process shall cover to the definition of several key planning and dimensioning parameters for 
minimizing the interference between both systems in the $900 \mathrm{MHz}$ frequency band and/or the establishment of a coordination measures between railway operators and public mobile broadband services operators that minimize the risk for harmful interferences [6].

For a complete interference analysis, it is necessary to cover several complex topics, such as, spectral re-growth, victim's receiver blocking response and distortion caused by intermodulation effects, and the specific high-speed railway propagation conditions [7]. The complexity of the interference analysis process, covering all these aforementioned topics, demand from the use of assessment software tools. There are several software tools for the interference assessment between wireless communication systems: SPECTRUM XXI, MSAM ('Microcomputer Spectrum Analysis') and SEAMCAT. However, all these tools lack in addressing some specificities of the high-speed railway environments, like specific signal propagation, or in the implementation of intermodulation products due to multiple transmitters.

To overcome these limitations, the authors present in this paper a novel software tool designed to evaluate the coexistence, in terms of interference, between public commercial communication systems: GSM, 3G, LTE and high-speed railway one, GSM-R. The developed tool allows for accurate interference analysis that will allow to take the required measures to avoid interferences.

The outline of this paper is as follows. Section two addresses the main factors which drive interference issues in railway scenarios. Section three focuses on the description of the developed software tool, devoting special attention to specific interference calculation mechanisms as well as railway specificities that have a key impact on the reliability of interference evaluation process. The fourth section presents a use case, where the coexistence between LTE and GSM-R is assessed for a high-speed railway environment.

\section{INTERFERENCE IN RAILWAY SCENARIOS}

GSM-R is the current communication system used in railway communications for voice and train control data. It is a sub-system of European Rail Traffic Management System (ERTMS), and it is based on GSM and EIRENE - MORANE specifications which guarantee performance at speeds up to $500 \mathrm{~km} / \mathrm{h}(310 \mathrm{mph})$, without any communication loss. It is critical to avoid radio communication failures, either by coverage problems or by interference ones, since these failures may lead to unnecessary train emergency stops.

ECC Report 162 proposes accepted coexistence scenarios between LTE/UMTS and GSM-R [8]. GSM-R interferences situations have been described by European Railway Associations, as mentioned in "Memorandum of Understanding (MoU)", which was written between the European Commission and CER-UIC-UNIFE-EIM- GSM-R Industry Group [1]. Studies about interference issues and possible mitigation measures have been carried out by several organizations $[2,6,9]$.

Interference issues may respond to several undesirable effects, such as, spectral re-growth, intermodulation effects or non-linearities in the transmitter. However, these effects are emphasized when communication systems are deployed in adjacent frequency bands next to GSM-R ones. GSM-R and its extended version, EGSM-R are deployed in frequency bands from 873 to $880 \mathrm{MHz}$ for uplink and from 918 up to $925 \mathrm{MHz}$ for downlink.

A new European regulation has released $900 \mathrm{MHz}$ frequency band for UMTS/HSPA systems, the so called refarming, as indicated in the Directive 2009/766/EC. In addition, the digital dividend process may allow to the deployment of LTE in the $800 \mathrm{MHz}$ band, which could affect to GSM-R uplink. This fact could produce interferences with GSM spectrum because it is located adjacent to public GSM-UMTS900 service providers. 


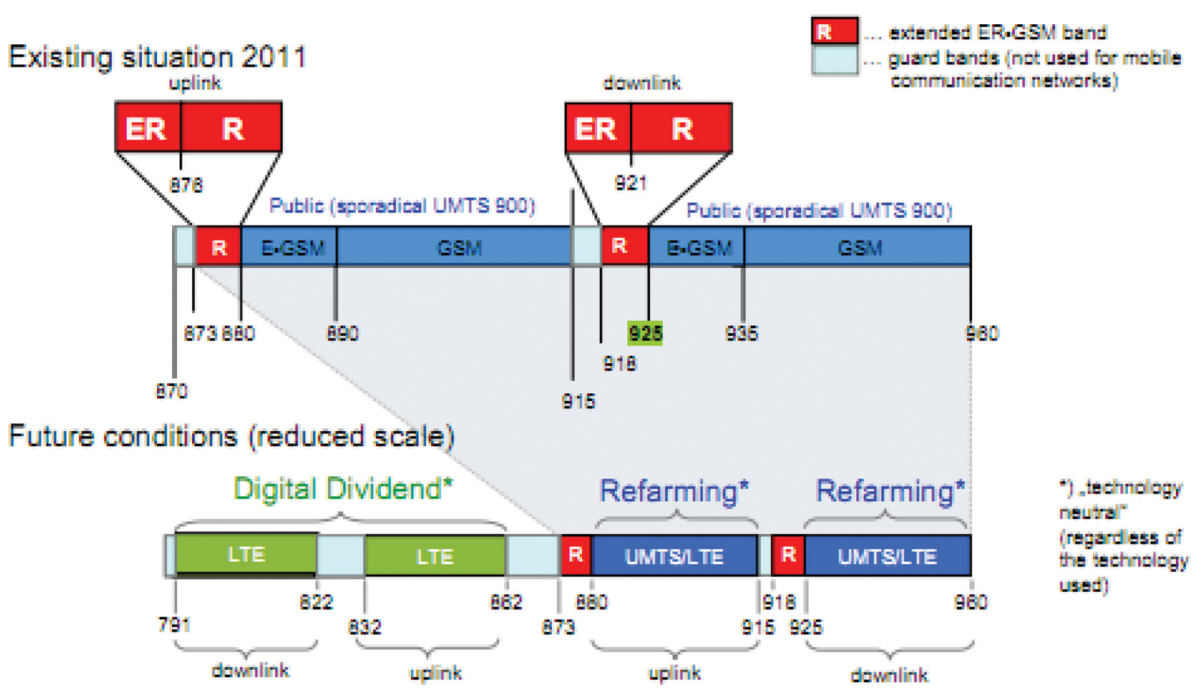

Figure 1: GSM-R frequency spectrum and digital dividend.

Nowadays, most of interferences cases in GSM-R networks are caused by blocking in GSM receivers and intermodulations in UMTS900 base stations. However, it is not possible to discard a future scenario where a new refarming process is made allowing the deployment of LTE in the $900 \mathrm{MHz}$ band. This conclusion could be applied to LTE 900 base stations, as shown in Fig. 1.

\section{SOFTWARE TOOL FOR RAILWAY INTERFERENCE ASSESSMENT}

In this chapter, the software tool for interference assessment in railway scenarios is presented. The underlying mechanisms of interference in wireless networks are also highlighted in this section and related to the developed software capabilities.

The reasons that motivated the development of this software have already been stated in the introduction section. Current interference analysis programs present some limitations that discourage their use in railway environments. The developed program allows calculating interferences due to unwanted emissions and blocking receiver, interference due to intermodulation of several transmitters, and spectral re-growth effects in broadband transmitters, as UMTS or LTE. The simulation methodology is based on Montecarlo simulations. For railways scenarios, it is considered that there is an interference issue when C/I relationship is lower than a predefined threshold.

Interferences in spread spectrum systems like UMTS are complex to be addressed. The implemented method in the software tool is known as jamming. Besides, it offers the possibility to define a receiver path, according to railway tracks, and it allows the selection of the most suitable railway propagation model, including tunnels. The block diagram of the developed software is shown in Fig. 2.

\subsection{Interferences due to intermodulation and harmonic distortion}

Spectral re-growth is an effect caused by distortion of nonlinear devices, for example, Power Amplifiers (PA). The effect of these non-linearities is closely related to the operating point of 


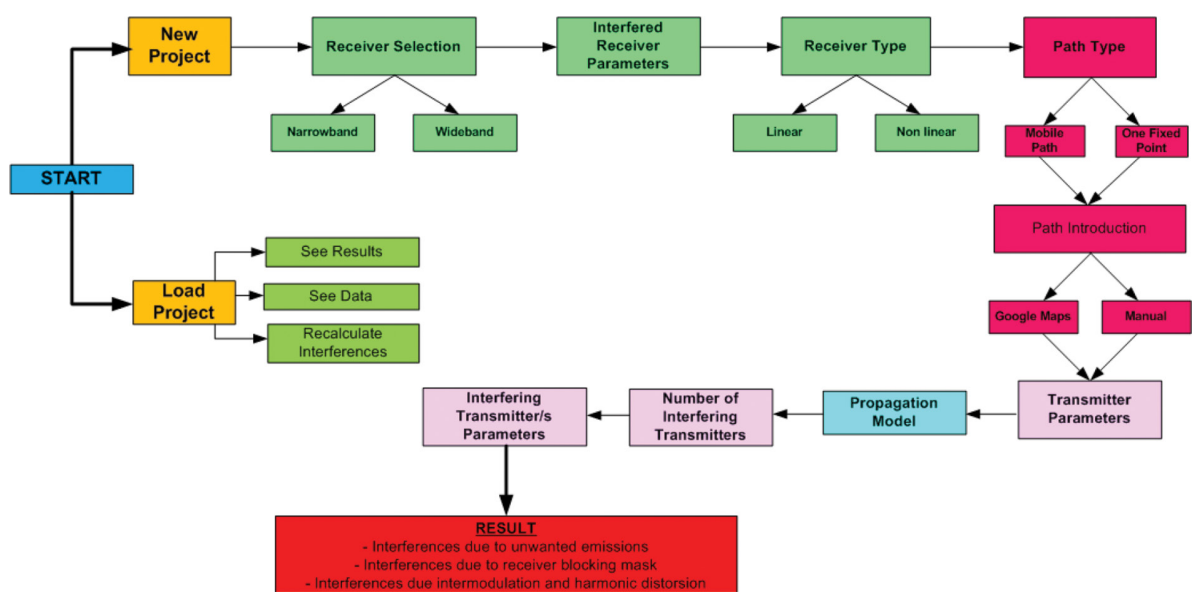

Figure 2: Block diagram of interference assessment software tool.

the PA, and the leakage into adjacent bands is mainly provoked by the Intermodulation Distortions (IMD). The developed software tool implements models to simulate real behavior of spectral re-growth in LTE due to IMD.

The multichannel intermodulation arises from signals generated by sweeping of different frequencies that pass through the same processed amplification chain in the transmitter. To calculate multichannel intermodulation with the developed software, it's necessary to do some simplifications. The program considers third order intermodulation, produced by sweeping of two or three independent signals with frequencies located near to the carrier frequency of transmitted channels, as shown in Fig. 3.

The intermodulation in narrowband systems is simulated assuming that carriers at transmitter output are not modulated and all channels have the same power and frequency separation.

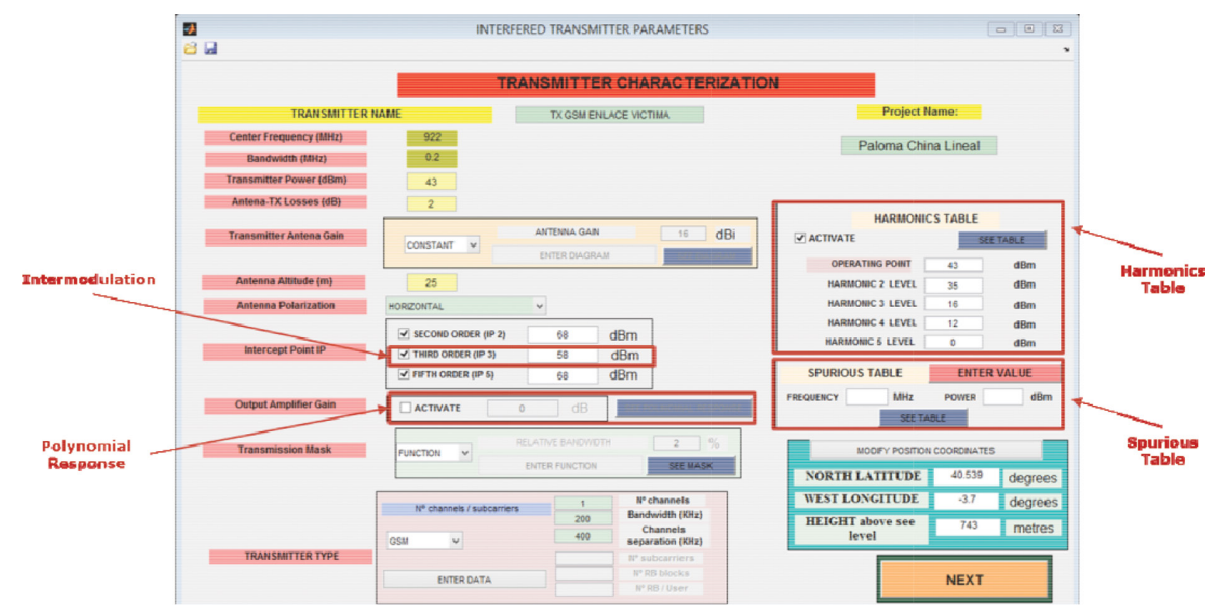

Figure 3: Transmitter characterization. 
The harmonic distortion interference, where transmitters have a powerful transmitter that works near to saturation point has also been considered. There are two methods to calculate harmonic distortion in the program: Harmonics table and Polynomial response. The harmonic distortion is introduced when it is analyzed the transmitter interference, as in Fig. 3.

\subsection{Interference due to unwanted emissions}

The 3GPP LTE standard specifications define two different kinds of unwanted emission: OutOf-Band (OOB) and spurious emissions. In LTE, OOB emissions are defined by means of Spectrum Emission Masks (SEMs) and/or Adjacent Channel Leakage Ratio (ACLR) requirements. The ACLR requirement is stricter than the SEM one. While the SEM measures the performance of the transmitter, the ACLR measures the power which actually leaks into certain specific nearby radio channels, which allows for assessing the interference levels due to unwanted emissions from LTE systems operating in adjacent frequency bands to GSM-R.

Interferences from unwanted emissions are due to imperfections in emissions masks of interfering transmitters. Some parameters are necessary to calculate them: interfering transmitter mask, receiver selectivity, the frequency difference between transmitter and receiver, transmission and reception antennas gain and propagation losses, as in Fig. 4.

\subsection{Interference due to receiver blocking mask}

Another important topic to be evaluated in the interferences analysis process is the receiver blocking response. It is defined as the maximum interfering signal level expressed in $\mathrm{dBm}$ which reduces the specified receiver sensitivity. The level of receiver blocking power is the level of power proceeding from the emission of the interfering transmitter captured by the receiver due to its selectivity. Interferences due to the interfered receiver blocking are generated due to receiver blocking mask, since it doesn't have the characteristics of a rectangular filter.

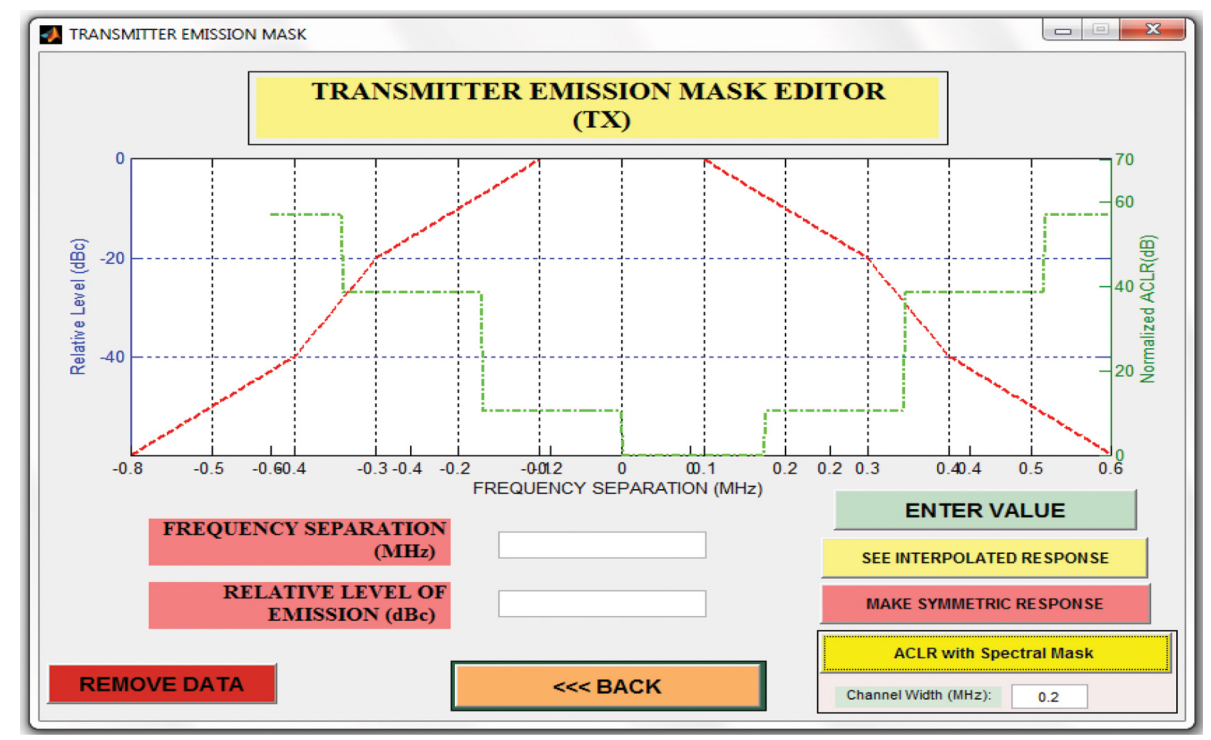

Figure 4: Transmitter emission mask editor. 
The interference analysis programs allow for the definition of receiver mask in several ways. By defining the receiver bandwidth as a percentage of central frequency, or by defining frequency response in the same way than the interfering transmitter mask.

\subsection{Radio propagation models in railway environments}

It is clear that the characterization of the transmitter and receiver has definitely an impact when assessing the previously defined interference requirements, and therefore, in the whole interference process. However, achieving accurate results in this process also requires from a proper and reliable characterization of the radio propagation conditions in the considered environments for both the desired link and the interferer one.

For modeling propagation in railway environments, it will be used the specific channel models for HSR scenarios described in [10]. It follows the methodology proposed by Winner II models, although it has not been standardized yet. An example of channel realization is shown in Fig. 5.

\section{CASE STUDY}

In this section, it will be assessed the theoretical performance of the developed software tool. The evaluation will be performed in a high-speed railway scenario, which can be seen in Fig. 6. In this scenario, both GSM-R and LTE communication systems coexist in the same location. GSM-R system is devoted to provide railway operational services, while the other serves on-board passengers. It is supposed that LTE is deployed in the $900 \mathrm{MHz}$ band, regarding the foreseen LTE refarming process.

It is defined a railway track trajectory about 1,000 meters in an open area railway scenario without obstacles. Both GSM-R and LTE remote radio heads are located on a mast which is

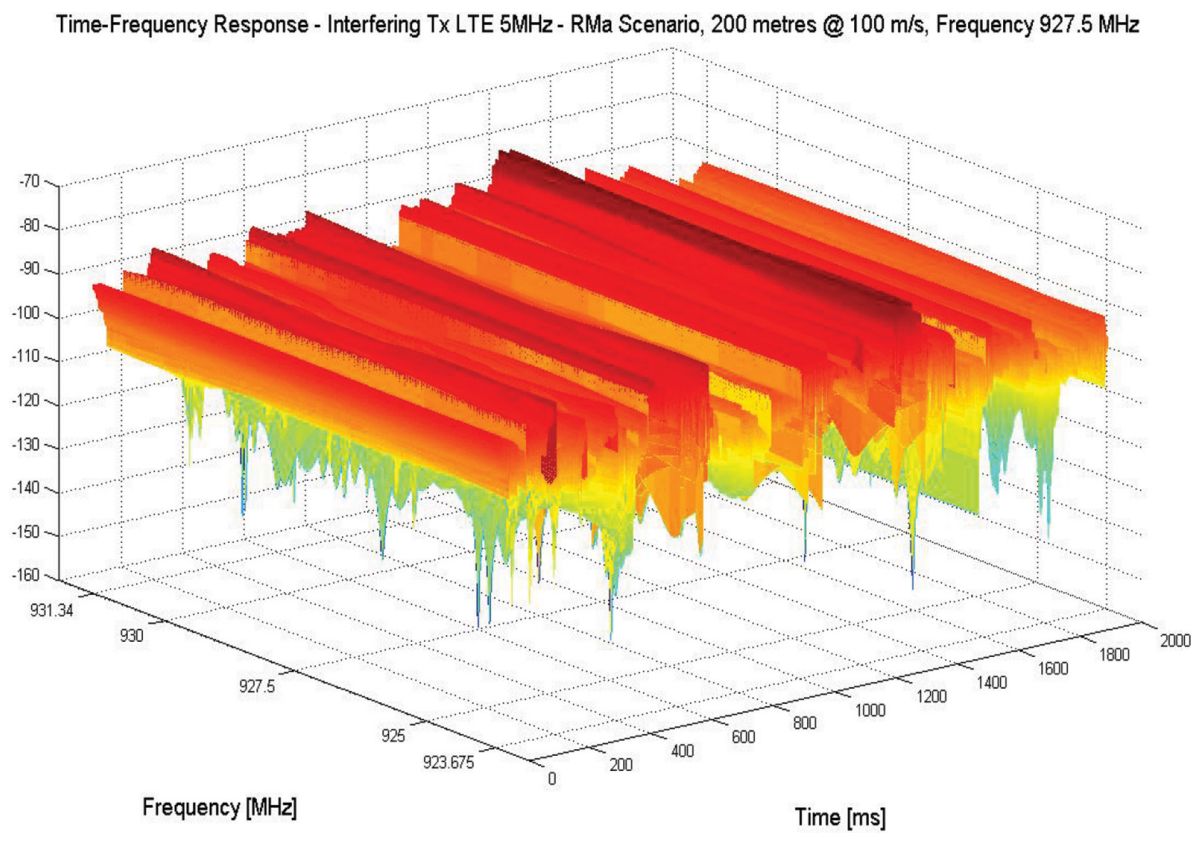

Figure 5: LTE channel realization in high-speed railway scenario. 


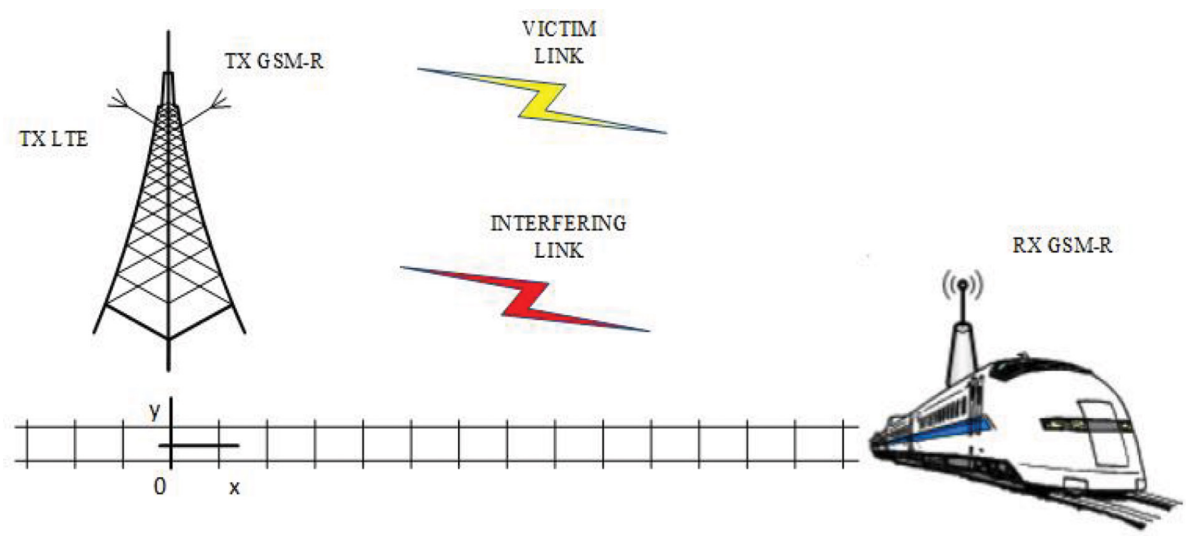

Figure 6: High-speed railway scenario for software evaluation purposes in the use case.

located 15 meters away from the tracks. It should be pointed out that the study has been developed for worst case, in which LTE antenna is directly pointing to the train. The simulation frequency band is the typical of GSM-R downlink, 921-925 MHz. LTE simulated channel is deployed in $927.5 \mathrm{MHz}$ with $5 \mathrm{MHz}$ bandwidth. For each iteration of Montecarlo series, specific GSM-R and LTE radio propagation channels are generated for an open space railway scenario. These complex time-frequency models takes into account the temporary variability due to shadowing or/and fast fading.

The train moves at $100 \mathrm{~m} / \mathrm{s}$ and C/I relation is evaluated every $1 \mathrm{~ms}$, or every $1 \mathrm{~cm}$ in the space domain. The considered threshold for interference is $12 \mathrm{~dB}$. 1,000 Montecarlo iterations are performed. A specific snapshot of one Montecarlo iteration is shown in Fig. 7.

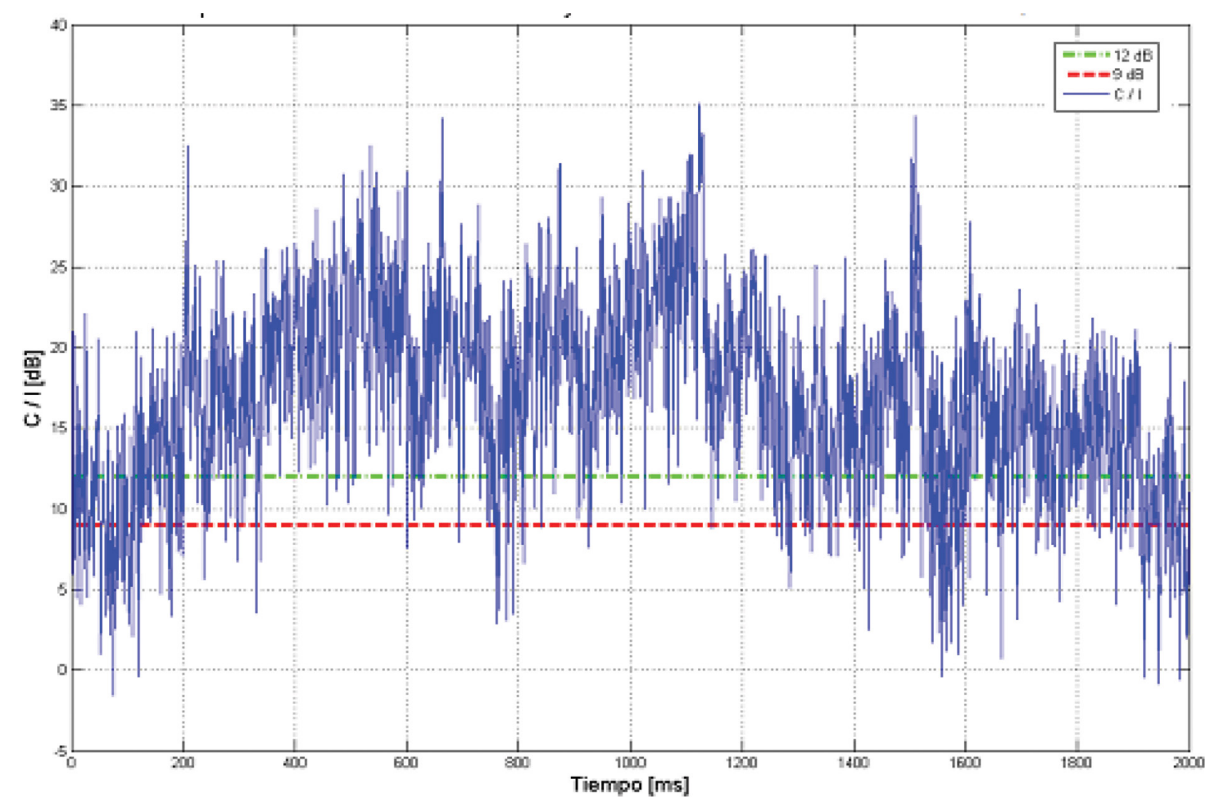

Figure 7: Snapshot of one Montecarlo iteration. Evaluation of C/I for GSM-R system in the described scenario. 
The final output provided by the software is the number of times that the C/I threshold level is not reached in the overall simulation process, expressed as a certain percentage. For this specific simulation process, the software tool provides a $17 \%$ of total interference issues regarding all simulation runs.

The close proximity between GSM-R and LTE transmitters and deployment channels may have a dramatic impact on GSM-R performance. The program also provides the minimum separation distance between the victim and the interferer systems for avoiding harmful interference effects. Other possible measures may be the modification of LTE transmitter masks or the installation of more selective receiver filters in the GSM-R system.

\section{CONCLUSIONS}

In this paper, a novel software tool designed for the evaluation of coexistence between highspeed railway communication systems and public commercial is presented.

The developed software allows for a realistic and accurate zero on-site evaluation process. The results provided by the program allow taking measures for ensuring coexistence if needed. These measures may lead to the avoidance of potential harmful interference effects in advance, thus reducing the system deployment costs.

\section{ACKNOWLEDGMENTS}

This work is supported by the Spanish Ministry of Economy and Competitiveness within the projects IPT-2011-1272-430000, IPT-2011-1034-37000 and TEC2014-53815-R.

\section{REFERENCES}

[1] Memorandum of Understanding (MoU) between the European Commission, the European Railway Agency and the European Rail sector Associations (CER-UIC-UNIFEEIM-GSM-R Industry Group-ERFA) concerning the strengthening of cooperation for the management of ERTMS.

[2] Co-Existence of UMTS900 and GSM-R systems. Omnitele Whitepaper. 30 August 2011.

[3] Barnard, A., "UMTS900 - GSM-R Interference Measurements”, OFCOM, June 2011.

[4] Calle-Sanchez, J., Molina-Garcia, M., Alonso, J.I. \& Fernandez-Durán, A., Long term evolution in high speed railway environments: feasibility and challenges. Bell Labs Technical Journal, 18(2), pp. 237-253, 2013.

http://dx.doi.org/10.1002/bltj.21615

[5] UIC WG FM, "Report On Interferences to GSM-R”. Version 2.0, 2012.

[6] "Coexistence between GSM-R and 3G/4G Systems in the $900 \mathrm{MHz}$ Frequency Band Swedish View", Trafikverket, March 2013.

[7] Briso-Rodriguez, C., López, C.F., Fernández, J.R.O., Pérez, S., Draskovic, D., CalleSánchez, J., Molina-Garcia, M., Alonso, J.I., Rodríguez, C., Hernández, C., Moreno, J., Rodríguez-Piñeiro, J., García-Naya, J.A., Castedo, L. \& Fernandez-Durán, A., Broadband access in complex environments: LTE on railway. The Institute of Electronics, Information and Communication Engineers (IEICE), E97-B(8), pp, 1514-1527, 2014.

[8] ECC Report \#162, "Practical Mechanism to improve the compatibility between GSM-R public mobile networks and guidance on practical coordination". May 2011.

[9] "Interferences into GSM-R due to public mobile radio networks", International Union of Railways (UIC), March 2011.

[10] China (People's Republic of), "Supporting information towards propagation prediction method for high-speed train wireless communication systems", UIT Tech. Doc. UIT-R WP3K-AR, August 2013. 